Präv Gesundheitsf 2022 · 17:428-434 https://doi.org/10.1007/s11553-021-00898-x Eingegangen: 12. April 2021

Angenommen: 5. August 2021

Online publiziert: 15 . September 2021

(c) Der/die Autor(en) 2021
Kathrin Allgayer ${ }^{1}$ - Carolin Bäßler ${ }^{1} \cdot$ Regina Jutz ${ }^{2}$ Marlen Niederberger ${ }^{1}$ (D)

'Forschungsmethoden in der Gesundheitsförderung und Prävention, Pädagogische Hochschule Schwäbisch Gmünd, Schwäbisch Gmünd, Deutschland

${ }^{2}$ Abteilung Soziologie, Pädagogische Hochschule Schwäbisch Gmünd, Schwäbisch Gmünd, Deutschland

\title{
Hochschulbeschäftigte in der Coronapandemie
}

\section{Auswirkungen auf die Gesundheit und das Wohlbefinden von Hochschulbeschäftigten mit Kind(ern) im Kita- und Grundschulalter}

\begin{abstract}
Die Coronapandemie und deren Schutzmaßnahmen bestimmen in den Jahren 2020/21 das öffentliche und private Leben in Deutschland. Durch die Schließungen von Kitas und Schulen sind insbesondere berufstätige Eltern einer Mehrbelastung im Alltag ausgesetzt [8]. Berufliche Rahmenbedingungen können diese abfangen oder verstärken [4]. In der vorliegenden Studie werden die Auswirkungen der Coronapandemie auf die Gesundheit und das Wohlbefinden von Hochschulbeschäftigten mit Kind(ern) im Kitaund Grundschulalter vorgestellt.
\end{abstract}

\section{Hintergrund}

Die Coronapandemie erweist sich 2020/21 für die gesamte deutsche Bevölkerung als multidimensionaler Stressfaktor, welcher auch negative Auswirkungen auf die Gesundheit derer hat, die nicht an COVID-19 („,coronavirus disease 2019“) erkranken [12]. Sie gehen u.a. aus Einschränkungen sozialer Kontakte, geringem Kontrollerleben sowie geringer Selbstwirksamkeitserwartung hervor [2]. Belastungen entstehen auch aufgrund notwendiger Schutzmaßnahmen und Regelungen im Arbeitskontext [13]. Diese Maßnahmen können sekundäre Gefährdungen hervorrufen, was für die Themen Homeoffice/Telearbeit [15] mit zunehmender Entgrenzung von Arbeit und Familie belegt ist [3].
Befunde zeigen, dass coronabedingte Veränderungen insbesondere für berufstätige Eltern herausfordernd sind. Vor allem während der zwei Lockdowns (03.-06.2020 und 12.2020-02.2021), in denen bundesweit Schulen und Kitas geschlossen wurden, waren Eltern einer Mehrbelastung im Alltag ausgesetzt und gezwungen, bspw. durch Homeschooling zusätzliche Aufgaben zu bewältigen [2, 10]. Diese Belastungsfaktoren erzeugen nach bisherigen Befunden verschiedene psychische, soziale und physische Gefährdungen [20]. Eltern mit Kindern unter 6 Jahren erleben in der Coronapandemie verglichen mit Eltern älterer Kinder einen stärkeren Rückgang der allgemeinen Lebenszufriedenheit [8]. Die Situation erscheint für Beschäftigte in nicht-systemrelevanten Berufen belastend, weil sie in den meisten Bundesländern im ersten Lockdown keinen und im zweiten Lockdown nur unter bestimmten Bedingungen einen Anspruch auf eine Notbetreuung hatten [10].

\section{Corona und Hochschul- beschäftigte}

Internationale und nationale Studien belegen Folgen der Coronapandemie auf die Arbeitsproduktivität, die Arbeitsplatzgestaltung und die Gesundheit der Hochschulbeschäftigten $[16,24]$. So zeigt eine repräsentative Studie aus Großbritannien, dass $66 \%$ in dieser Zeit ein hohes Stresslevel angeben [25]. Auch an einer
Berliner Hochschule geben $71 \%$ der Lehrenden im Sommersemester 2020 an, einer starken allgemeinen Belastung ausgesetzt zu sein [1]. Inwieweit sich diese Folgen insbesondere auf Hochschulbeschäftigte mit Kind(ern) auswirken, wird im vorliegenden Forschungsprojekt untersucht. Dem ganzheitlichen Gesundheitsverständnis der Weltgesundheitsorganisation folgend, werden dabei soziale, physische und psychische Aspekte erhoben [26].

\section{Methodik}

\section{Studiendesign}

Die Studie wurde als quantitative Online-Befragung konzipiert und durch vier Strategien entwickelt:

1. Nutzung etablierter Items zur Messung von Gesundheit und Wohlbefinden: WHO (fünf)-Fragebogen zum Wohlbefinden [17], sozioökonomisches Panel [9], Fragebogen zum aktuellen körperlichen Wohlbefinden [6] sowie zum allgemeinen habituellen Wohlbefinden [27].

2. Sichtung aktueller Studien zur Gesundheit von Beschäftigten in der Coronapandemie,

3. Durchführung fünf qualitativer Expert*inneninterviews aus dem Kontext Hochschule,

4. partizipative Entwicklung des Fragebogens mit Praxispartner*innen aus den Bereichen Gleichstellung, Ge- 
Tab. 1 Deskriptive Beschreibung der Be-

fragten (Anteile in Klammern; $n=1104$ )

Bundesland

Baden-Würt- Sachsen temberg (34\%)

(66\%) $\quad n=373$

$n=731$

\section{Geschlecht:}

Männlich

$241(33,0 \%) \quad 148$

$(39,7 \%)$

Weiblich

$488(66,8 \%) \quad 224$

$(60,1 \%)$

Divers $\quad 2(0,3 \%) \quad 1(0,3 \%)$

Kinderanzahl:

Ein Kind

$343(46,9 \%) \quad 182$

$(48,8 \%)$

Zwei Kinder $\quad 325(44,5 \%) \quad 171$

$(45,8 \%)$

Drei oder mehr $63(8,6 \%) \quad 20$

Kinder

$(5,4 \%)$

Arbeitsverhältnis:

Vollzeit

$328(45,4 \%) \quad 216$

$(58,1 \%)$

Teilzeit

$395(54,6 \%) \quad 156$

(41,9\%)

Befristet

$290(40,1 \%) \quad 210$

$(56,5 \%)$

Unbefristet

$434(59,9 \%) \quad 162$

$(43,5 \%)$

Beschäftigungsgruppe:

\begin{tabular}{|c|c|c|}
\hline $\begin{array}{l}\text { Wissenschaft/ } \\
\text { Lehre }\end{array}$ & $404(55,5 \%)$ & $\begin{array}{l}232 \\
(62,4 \%)\end{array}$ \\
\hline $\begin{array}{l}\text { Technik/ } \\
\text { Verwaltung/ } \\
\text { Wissenschafts- } \\
\text { management }\end{array}$ & $321(44,1 \%)$ & $\begin{array}{l}132 \\
(35,5 \%)\end{array}$ \\
\hline Sonstiges & $3(0,4 \%)$ & $8(2,2 \%)$ \\
\hline $\begin{array}{l}\text { Mindestens } 50 \% \\
\text { der Zeit im Home- } \\
\text { Office: }\end{array}$ & $588(83,3 \%)$ & $\begin{array}{l}282 \\
(78,6 \%)\end{array}$ \\
\hline
\end{tabular}

sundheitsmanagement und Arbeitsschutz an Hochschulen aus Sachsen (SN) und Baden-Württemberg (BW).

\section{Stichprobe}

Zielgruppe sind Hochschulbeschäftigte mit Kind(ern) im Kita- und Grundschulalter in SN und BW. Die Auswahl der Bundesländer erfolgte aufgrund eines angestrebten Ost-West-Vergleichs, kombiniert mit hohen Inzidenzwerten im Laufe der Coronapandemie 2020. Zudem bildeten BW und SN 2019 die Schlusslichter im Länderranking nach Gleichstellungsaspekten an Hochschulen [14]. Die vergleichsweise niedrigen
Anteile an weiblichen Promovierenden und Beschäftigten deuten strukturelle Probleme an, die bereits vor der Coronapandemie existierten, sich aber im Zuge der coronabedingten Herausforderungen als ungünstige Voraussetzung möglicherweise potenzieren.

Die Befragung fand online während des zweiten Lockdowns (11.01.2021 bis 07.02.2021) statt. Die Stichprobe beruht auf einem Convenience-Sampling, d.h. die Hochschulbeschäftigten wurden über Gatekeeper angesprochen. Als Gatekeeper fungierten verschiedene Praxispartner*innen auf der Landesebene aus den Bereichen Gleichstellung und betriebliches Gesundheitsmanagement im Kontext Hochschule, die über E-MailVerteiler der assoziierten Stellen an den Hochschulen verfügten. Zusätzlich wurden E-Mails an die Rektor*innen aus BW und SN versendet, mit der Bitte zur Verteilung an die Beschäftigten. Auf diese Weise wurden die Beschäftigten über mehrere Kanäle über die Befragung informiert und gebeten, sich $\mathrm{zu}$ beteiligen. Allerdings konnte bei dieser Vorgehensweise die Grundgesamtheit der Stichprobe nicht bestimmt und somit die Rücklaufquote nicht berechnet werden.

Insgesamt haben sich 1258 Hochschulbeschäftigte aus BW oder SN an der Befragung beteiligt. Nach einer Bereinigung des Datensatz, bei der nur Befragte berücksichtigt werden, die mindestens 75\% des Fragebogens ausgefüllt haben, verblieben 1104 Teilnehmende. Die Beschreibung der Stichprobe ist in - Tab. 1 dargestellt.

\section{Auswertung}

Die folgende deskriptive Auswertung stellt die Gesundheit und das Wohlbefinden der Befragten während der Coronapandemie dar. Bei allen Fragen wurden die Befragten gebeten, die Zeit der Coronapandemie von März 2020 bis zum Befragungszeitpunkt zu berücksichtigen. Das subjektive Wohlbefinden wurde als Mittelwertindex aus 8 Items zu psychischen, sozialen und physischen Aspekten berechnet. Der Index nimmt Werte zwischen 0 (geringstes) und 5 (höchstes) Wohlbefinden an. Aufgrund abweichender Berechnungen können keine Vergleiche mit etablierten Skalen vorgenommen werden. Anhand des nicht-parametrischen Mann-WhitneyU-Tests wurde untersucht, ob es zwischen den Befragten in SN und BW signifikante Unterschiede gibt. Dieser Fokus wurde gewählt, um einen explorativ-deskriptiven Eindruck möglicher Ost-West-Unterschiede zu erhalten.

\section{Ergebnisse}

\section{Allgemeine Lebensumstände in der Coronapandemie}

Die meisten Befragten geben an, dass sich ihre soziale Situation (72\%) und die Balance zwischen Privat- und Berufsleben $(76 \%)$ während der Coronapandemie verschlechtert hat. Weniger kritisch werden die finanzielle Situation oder das Verhältnis zu dem Kind/den Kindern angesehen (•Abb. 1). $64 \%$ haben meistens oder die ganze Zeit jemanden, mit dem sie über alles reden können. Insgesamt zeigen sich bei der Beurteilung der Lebenssituation keine signifikanten Unterschiede zwischen den Bundesländern.

Die Befragten beurteilen ihre Gesundheit $\mathrm{zu} 14 \%$ als sehr gut, $39 \%$ als gut, $26 \%$ als zufriedenstellend, $18 \%$ als weniger gut und $3 \%$ als schlecht. Der Mittelwert der Bundesländer ist identisch bei 2,6 ( $S D=1,0 ;$ gut bis zufriedenstellend) auf einer Skala von 1 (sehr gut) bis 5 (schlecht).

Auffällig ist hier die ungleiche Verteilung von Gesundheit zwischen den Geschlechtern: Mütter $(M D=2 ; M W=2,62$; $S D=1,01)$ geben einen signifikant $(U=126.301,50 ; p<0,05)$ schlechteren Gesundheitszustand an als Väter $(M D=2$; $M W=2,47, S D=1,05)$. Auch der Index zum subjektiven Wohlbefinden fällt für Mütter $(M D=2 ; M W=2,44 ; S D=0,92)$ signifikant $\quad(U=128.113,5 ; \quad p<0,05)$ geringer aus als für Väter $(M D=2$; $M W=2,58 ; S D=0,97$ ).

Für die beiden Bundesländer zeigt der Index zum subjektiven Wohlbefinden geringe Unterschiede: in beiden Bundesländern markieren $51 \%$ der Befragten Antwortkategorien, die auf ein mangelndes Wohlbefinden schließen lassen. Für SN ergibt sich ein Mittel- 
Präv Gesundheitsf 2022 · 17:428-434 https://doi.org/10.1007/s11553-021-00898-x

(c) Der/die Autor(en) 2021

\section{K. Allgayer · C. Bäßler · R. Jutz · M. Niederberger}

\section{Hochschulbeschäftigte in der Coronapandemie. Auswirkungen auf die Gesundheit und das Wohlbefinden von Hochschulbeschäftigten mit Kind(ern) im Kita- und Grundschulalter}

\section{Zusammenfassung}

Hintergrund. Die Coronapandemie erweist sich 2020/21 für die gesamte deutsche Bevölkerung als multidimensionaler Stressfaktor. Erste Studien deuten an, dass diese Zeit insbesondere für berufstätige Eltern mit Kind(ern) herausfordernd ist. Sie sind Belastungsfaktoren ausgesetzt, aus denen sich psychische, soziale und physische Gefährdungen ergeben können.

Ziel der Arbeit. Untersucht werden die Auswirkungen der Coronapandemie auf die Gesundheit und das Wohlbefinden von Hochschulbeschäftigten mit Kind(ern) im Kitaund Grundschulalter aus Sachsen (SN) und Baden-Württemberg (BW).

Methodik. Mittels eines standardisierten Online-Fragebogens wurden Hochschulbe- schäftigte in SN und BW zu ihrer Lebens- und Arbeitssituation in der Coronapandemie sowie ihrem subjektiven Wohlbefinden und ihrer Gesundheit befragt.

Ergebnisse. Besonders belastend nehmen die Befragten die Veränderung der sozialen Situation, die Verschlechterung der Balance zwischen Berufs- und Privatleben und das Verschwimmen der Grenzen zwischen Arbeits- und privater Zeit wahr. Die befragten Hochschulbeschäftigten in SN zeigen bei verschiedenen Belastungsfaktoren in Bezug auf die Arbeitssituation signifikant schlechtere Bewertungen als die Befragten in BW. In beiden Bundesländern geben jeweils mehr als die Hälfte der Befragten eher kritische Werte für ihr Wohlbefinden an.
Schlussfolgerung. Die Coronapandemie und die damit einhergehenden Schutzmaßnahmen haben negative Auswirkungen auf das Wohlbefinden von Hochschulbeschäftigten mit Kind(ern) im Kita- und Grundschulalter. Inwieweit sich diese Effekte längerfristig zeigen, wenn beispielsweise strukturelle Maßnahmen im Bereich Homeoffice und Online-Lehre in Hochschulen verstetigt werden, ist zu prüfen.

Schlüsselwörter Hochschule · COVID-19 - Lebenszufriedenheit . Online-Befragung $\cdot$ Wissenschaftler

\section{University employees during the coronavirus pandemic. Health and well-being of university employees with daycare- and elementary school-aged children}

\section{Abstract}

Background. The coronavirus pandemic turns out to be a multidimensional stressor for the entire German population in 2020/2021. Studies indicate that these corona-related changes are also challenging for university employees with children. Parents were exposed to stress factors, which can be negatively associated with various psychological, social and physical aspects.

Objectives. Investigation of the impact of the coronavirus pandemic on health and wellbeing of university employees with daycareand primary school-aged children.

Methods. Using a standardized online

questionnaire, university employees in Saxony and Baden-Wuerttemberg were contacted and asked about their living and working situation during the coronavirus pandemic as well as their subjective well-being and health. Results. The respondents perceive the changes in the social situation, the deterioration of the balance between work and private life and the blurring of boundaries between work and private time as particularly stressful. The respondents in Saxony show significantly worse ratings than in Baden-Wuerttemberg for various stress factors in relation to the work situation. In both states, slightly more than half of the respondents marked categories that indicate a lack of well-being.
Conclusions. The coronavirus pandemic and its accompanying protective measures have had negative effects on the well-being of university employees with children of daycare and elementary school age. The extent to which these effects will become apparent in the longer term, for example, whether structural measures of remote work and online teaching in universities are made permanent, must be examined in the future.

\section{Keywords}

University · COVID-19 · Life satisfaction · Online survey $\cdot$ Scientist wert von 2,47 $(S D=0,88)$ und für BW von $2,5(S D=0,97)$ aus dem Wertebereich von 0 (geringstes) bis 5 (höchstes) Wohlbefinden.

Die Ergebnisse zu den Beanspruchungen im Arbeitskontext zeigen, dass $60 \%$ der Befragten eher bzw. voll und ganz zustimmen, sich ständig gehetzt sowie unter Druck zu fühlen und nicht zur Ruhe zu kommen (•Abb. 2). Fast die Hälfte der Befragten erlebt vermehrt körperliche Beschwerden.
Signifikante Unterschiede zeigen, dass die Hochschulbeschäftigten in SN häufiger Probleme haben, ihre Arbeit zu bewältigen $(U=124.443, p<0,05 ; \mathrm{SN}$ : $n=373 ; M D=3 ; M W=2,77 ; S D=1,131$; BW : $n=728 ; \quad M D=3 ; \quad M W=2,94$; $S D=1,139)$ und gedanklich weniger vom Arbeitstag abschalten können $(U=11.774 ; p<0,01 ; \mathrm{BW}: n=729 ; M D=3$, $M W=3,16 ; \quad S D=1,152 ; \quad \mathrm{SN}: \quad n=371 ;$ $M D=4 ; M W=3,43 ; S D=1,13)$ als die Befragten in BW.

\section{Hochschulen in der Corona- pandemie}

Die Frage nach Belastungsfaktoren in Bezug auf die Arbeitssituation zeigt, dass die Hochschulbeschäftigten in BW bei 8 von 12 Items eine signifikant bessere Bewertung angeben als in SN. Dies äußert sich bei der Planung des Arbeitstags, der Unterstützung bei digitalen Tools, der Veränderung von Arbeitsabläufen, Verantwortungsbereichen in der Hochschule, Unterstützung durch die Führung, er- 


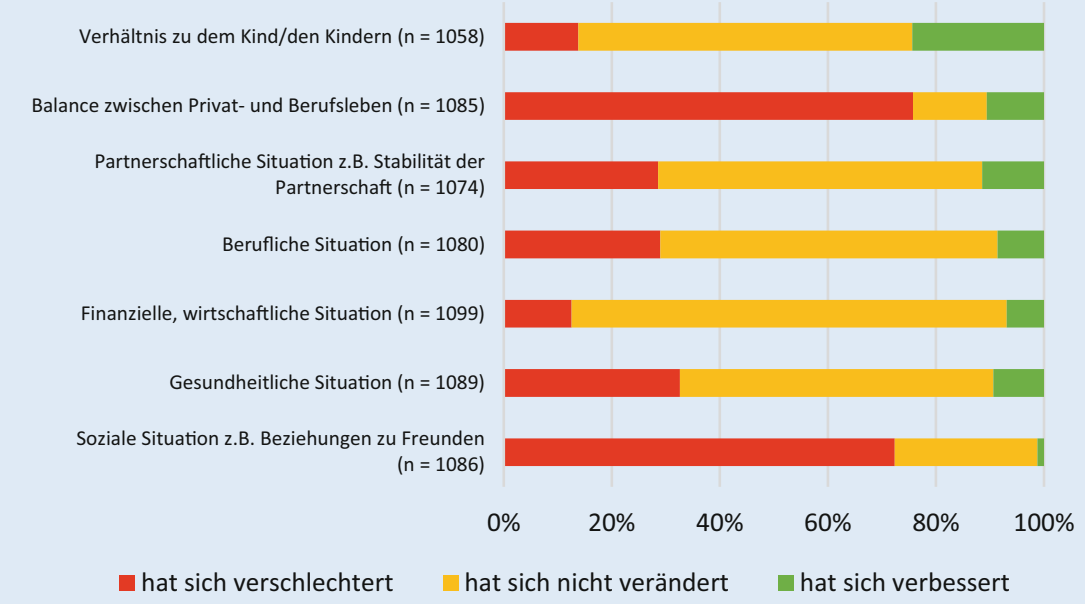

Abb. 1 \ Wie hat sich Ihre Lebenssituation in der Coronapandemie im Vergleich zur Situation vorher verändert?

gonomischer Arbeitsplatzgestaltung und beruflicher Überforderung. Der größte Belastungsfaktor in beiden Bundesländern stellt mit $75 \%$ Zustimmung das Verschwimmen der Grenzen zwischen Arbeits- und privater Zeit dar. Hier fällt die Einschätzung in SN signifikant schlechter aus.

Die Befragten in SN empfinden ihre Arbeitszeit sowie den -aufwand als signifikant länger bzw. mehr als die Befragten in BW. Es bestehen keine signifikanten Unterschiede bei der Angst sich mit Corona anzustecken, beim sozialen Umgang sowie der fachlichen Unterstützung durch Kolleg*innen (•Tab.2).

Die Arbeitssituation unter Coronabedingungen ist für $75 \%$ der Befragten sinnvoll, $82 \%$ verständlich sowie $66 \%$ handhabbar (stimme voll und ganz zu und stimme eher $z u$ ). Der Mittelwertindex zur Resilienz aus diesen 3 Items zeigt bei den Befragten in BW signifikant $(U=117.990,5 ; p<0,001)$ höhere Werte $(n=723 ; M D=4 ; M W=4,11 ; S D=1,055)$ als in $\mathrm{SN}(n=371 ; M D=4 ; M W=3,98$; $S D=1,064)$.

Mit den Arbeitsbedingungen zu Hause sind 39\% aller Befragten sehr bzw. eher unzufrieden. Dabei sind Befragte in SN $(n=366 ; M D=3 ; M W=3,25 ; S D=1,166)$ signifikant $(U=103.822,5 ; p<0,001)$ unzufriedener als in $\mathrm{BW}(n=700 ; M D=3$; $M W=2,86 ; S D=1,130) .46 \%$ der Befragten erleben gesundheitliche Belastungen durch die Arbeitsbedingungen zu Hause.
Auf die Frage nach Unterstützungsangeboten durch die Hochschule wünschen sich etwa $44 \%$ von der Hochschulleitung, dass die Situation von Eltern mehr thematisiert wird. Danach folgt der Wunsch, zu Hause bleiben zu können, wenn ein Kind in Quarantäne muss und finanzielle Mittel für die Arbeitsplatzausstattung zu Hause.

Zudem wurde nach der Zufriedenheit der Unterstützungsleistungen verschiedener Hochschulakteure gefragt; die Befragten in $\mathrm{SN}$ fühlen sich durch diese signifikant $(p<0,05)$ weniger unterstützt als in BW (• Abb. 3). Die Nachfrage nach notwendigen Veränderungen von Rahmenbedingungen für Beschäftigte mit Kind(ern) im Kita- und Grundschulalter, auch nach der Coronapandemie, bejahten mehr als $80 \%$ der Beschäftigten beider Bundesländer.

\section{Diskussion}

Im Hinblick auf die Gesundheit und das Wohlbefinden von Hochschulbeschäftigten stellt sich insbesondere das Thema Entgrenzung als herausfordernd heraus. Politik und Hochschulen sind in der Verantwortung, Beschäftigte mit Kind(ern) bei der Vereinbarkeit von Familie und Beruf in und nach der Coronapandemie zu unterstützen und mögliche Bewältigungsstrategien umzusetzen. Mit den in der Coronapandemie umgesetzten Schutzmaßnahmen konnten Beschäftig- te ihren Aufgaben als Arbeitsnehmer*in und Elternteil oft nicht gleichermaßen gerecht werden [5]. Zwar belegen frühere Studien und Metanalysen zu den Folgen von flexiblen Arbeitszeitmodellen, wie bspw. Homeoffice, verschiedene positive Effekte auf die Lebensqualität und die Vereinbarkeit von Familie und Beruf $[7,22]$, doch zu vermuten ist, dass es durch die zusätzlichen Herausforderungen der Pandemie wie Homeschooling und soziale Isolation zu einer Kumulation von Belastungen kommen kann, die kritisch zu reflektieren sind. Dies deuten die Befunde zu den Geschlechtsunterschieden an. Weitere Studienergebnisse sprechen ebenfalls dafür, dass Mütter während den Kita- und Schulschließungen überwiegend die Kinderbetreuung übernommen und damit die Hauptlast getragen haben. Bereits zuvor bestehende geschlechterspezifische Rollenund Arbeitsverteilungen von Müttern und Vätern wurden dadurch verstärkt [11]. Das mehrdimensionale Konstrukt des Wohlbefindens sowie die subjektive Gesundheit bewerten die befragten Mütter in der vorliegenden Studie deutlich kritischer als die Väter.

Die Befunde zeigen, dass es wichtig ist, eine hohe Transparenz von Coronainformationen für alle Hochschulmitglieder zu gewährleisten und insbesondere Hochschulleitungen für die Situation von Beschäftigten mit Kind(ern) im Kitaund Grundschulalter zu sensibilisieren. $\mathrm{Zu}$ ihrem Aufgabenbereich gehört die Durchführung regelmäßiger psychischer Gefährdungsbeurteilungen, um frühzeitig notwendige Interventionen einzuleiten [5]. Dies sollte auch in Ausnahmesituationen erfolgen.

Die durchgeführte Studie zeigt, dass die Coronapandemie negative Auswirkungen auf das Wohlbefinden der Hochschulbeschäftigten mit Kind(ern) in SN und BW hat. Die Befragten in BW bewerten ihre Arbeitssituation sowohl in der Hochschule als auch zu Hause in vielen Aspekten positiver als in SN. Möglicherweise werden die unterschiedlichen Bewertungen durch unterschiedliche Strukturen, Ressourcen und Erfahrungen im Bereich des betrieblichen Gesundheitsmanagements oder coronabedingt durch die jeweilige Inzidenzlage sowie die po- 


\section{Originalarbeit}
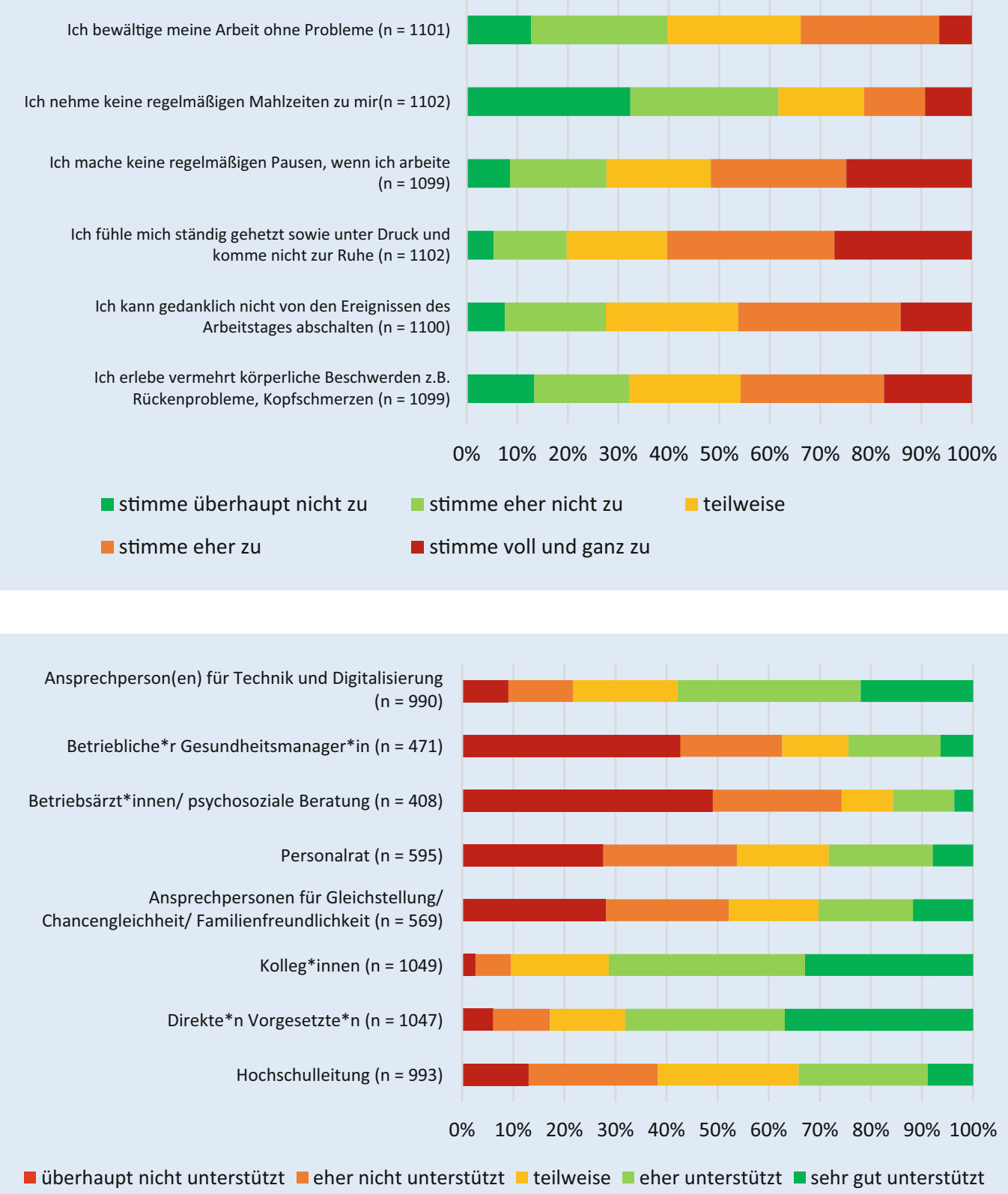

Abb. $2 \triangleleft$ Inwieweit stimmen Sie den folgenden Aussagen in Bezug auf Ihre Berufstätigkeit in der Zeit der Coronapandemie zu oder nicht zu?
Abb. $3<$ Wie bewerten Sie als Beschäftigte*r mit Kind(ern) im Kita- und Grundschulalter die Unterstützungsleistungen verschiedener Akteur*innen an Ihrer Hochschule in der Coronapandemie? litischen Regelungen innerhalb der Bundesländer beeinflusst. In der zweiten Coronawelle wies SN ab November 2020 eine durchgehend höhere Inzidenzrate auf als BW [18]. Der Anteil der befristet Beschäftigten in unserer Studie ist in SN um etwa 16 Prozentpunkte größer als in BW. Die höhere Befristungsquote könnte zu dem höheren Belastungsempfinden in SN führen, da die Beschäftigten ihre Arbeitsplatzsicherheit eventuell durch ein entsprechendes berufliches Engagement gewährleisten wollen. Zudem bestand für Hochschulbeschäftigte in SN bei fehlender Systemrelevanz beider Elternteile kein Anspruch auf eine Notbetreuung [19]. In BW wurde dieser Anspruch im zweiten Lockdown wesentlich niederschwelliger ermöglicht [21]. Generell arbeiten Mütter in Ostdeutschland mehr in Vollzeitstellen als in Westdeutschland [23], was auch die vorliegende Studie bestätigt. Dies könnte sich auch auf das Belastungsempfinden in der Krise auswirken. Diese Unterschiede stellten Hochschulbeschäftigte mit Kind(ern) in SN wohl vor größere Herausforderungen bei der Vereinbarkeit von Arbeit und
Kinderbetreuung als in BW. Weitere Analysen nach Geschlecht mit einem besonderen Fokus auf die vulnerable Gruppe von befristet beschäftigten Wissenschaftlerinnen mit Kind(ern) sind vorgesehen und sollen die Ergebnisse vervollständigen.

\section{Limitierung der Studie}

- Keine Repräsentativität für Hochschulbeschäftigte in BW und SN,

- keine Kontrollierbarkeit bei der Verteilung des Fragebogen-Links 


\begin{tabular}{|c|c|c|c|c|c|c|}
\hline & $\begin{array}{l}\text { Bundes- } \\
\text { land }\end{array}$ & $U$-Wert & $\begin{array}{l}\text { Signifikanzwert } \\
\text { (p) }\end{array}$ & $\begin{array}{l}\text { Median } \\
\text { (MD) }\end{array}$ & $\begin{array}{l}\text { Mittelwert } \\
\text { (MW) }\end{array}$ & $\begin{array}{l}\text { Standardabweichung } \\
\text { (SD) }\end{array}$ \\
\hline \multirow[t]{2}{*}{ Durch Corona ist mein Arbeitstag schwer planbar. } & BW (728) & \multirow[t]{2}{*}{$124.294,5$} & \multirow[t]{2}{*}{$0,021^{\mathrm{a}}$} & 4 & 3,41 & 1,223 \\
\hline & SN (372) & & & 4 & 3,6 & 1,148 \\
\hline \multirow{2}{*}{$\begin{array}{l}\text { Ich bekomme Unterstützung beim Erlernen und dem } \\
\text { Einsatz digitaler Tools für meine Tätigkeit. }\end{array}$} & BW (662) & \multirow[t]{2}{*}{$98.249,5$} & \multirow[t]{2}{*}{$0,002^{\mathrm{a}}$} & 3 & 3,25 & 1,221 \\
\hline & SN (337) & & & 3 & 3,01 & 1,171 \\
\hline \multirow{2}{*}{$\begin{array}{l}\text { Ich habe Angst, mich am Arbeitsplatz mit Corona } \\
\text { anzustecken. }\end{array}$} & BW (725) & \multirow[t]{2}{*}{$130.159,5$} & \multirow[t]{2}{*}{0,362} & 2 & 2,21 & 1,249 \\
\hline & SN (371) & & & 2 & 2,26 & 1,230 \\
\hline \multirow{2}{*}{$\begin{array}{l}\text { Seit Corona ist der Umgang mit meinen Kolleg*innen } \\
\text { weniger freundlich und unterstützend. }\end{array}$} & BW (700) & \multirow[t]{2}{*}{118.453} & \multirow[t]{2}{*}{0,165} & 2 & 1,99 & 1,125 \\
\hline & SN (356) & & & 2 & 2,06 & 1,081 \\
\hline \multirow[t]{2}{*}{ Seit Corona arbeite ich mehr und länger. } & BW (722) & \multirow[t]{2}{*}{113.414} & \multirow[t]{2}{*}{$<0,001^{b}$} & 3 & 3,03 & 1,310 \\
\hline & SN (356) & & & 2 & 2,69 & 1,228 \\
\hline \multirow{2}{*}{$\begin{array}{l}\text { Seit Corona haben sich meine Arbeitsabläufe negativ } \\
\text { verändert. }\end{array}$} & BW (722) & \multirow[t]{2}{*}{118.334} & \multirow[t]{2}{*}{$0,002^{\mathrm{a}}$} & 3 & 3,22 & 1,277 \\
\hline & SN (369) & & & 4 & 3,48 & 1,180 \\
\hline \multirow{2}{*}{$\begin{array}{l}\text { Seit Corona verschwimmen Grenzen zwischen Ar- } \\
\text { beitszeit und privater Zeit. }\end{array}$} & BW (725) & \multirow[t]{2}{*}{121.821} & \multirow[t]{2}{*}{$0,004^{\mathrm{a}}$} & 4 & 3,99 & 1,156 \\
\hline & SN (373) & & & 5 & 4,22 & 0,977 \\
\hline \multirow{2}{*}{$\begin{array}{l}\text { Seit Corona sind die Verantwortungs- und Aufgaben- } \\
\text { bereiche in der Hochschule unklar. }\end{array}$} & BW (677) & \multirow[t]{2}{*}{108.396} & \multirow[t]{2}{*}{$0,030^{\mathrm{a}}$} & 2 & 2,28 & 1,143 \\
\hline & SN (348) & & & 2 & 2,44 & 1,161 \\
\hline \multirow{2}{*}{$\begin{array}{l}\text { Seit Corona fehlt die soziale und fachliche Unterstüt- } \\
\text { zung durch Kolleg*innen. }\end{array}$} & BW (719) & \multirow[t]{2}{*}{129.961} & \multirow[t]{2}{*}{0,573} & 3 & 3,04 & 1,257 \\
\hline & SN (369) & & & 3 & 3,00 & 1,215 \\
\hline \multirow{2}{*}{$\begin{array}{l}\text { Seit Corona fehlt die Unterstützung und das Feed- } \\
\text { back durch die Führung. }\end{array}$} & BW (707) & \multirow[t]{2}{*}{$1.144 .001,5$} & \multirow[t]{2}{*}{$<0,001^{b}$} & 2 & 2,53 & 1,257 \\
\hline & SN (368) & & & 3 & 2,80 & 1,281 \\
\hline \multirow{2}{*}{$\begin{array}{l}\text { Seit Corona hat sich die ergonomische Arbeitsplatz- } \\
\text { gestaltung verschlechtert. }\end{array}$} & BW (719) & \multirow[t]{2}{*}{$121.073,5$} & \multirow[t]{2}{*}{$0,028^{a}$} & 3 & 3,10 & 1,430 \\
\hline & SN (366) & & & 4 & 3,30 & 1,425 \\
\hline Seit Corona fühle ich mich beruflich überfordert. & BW (721) & 121.361 & $0,011^{\mathrm{a}}$ & 2 & 2,21 & 1,105 \\
\hline & SN (370) & & & 2 & 2,36 & 1,051 \\
\hline $\begin{array}{l}\text { Absolute Zahlen in Klammern. MD }=1 \text {,stimme voll und } \\
\text { SN Sachsen, } B W \text { Baden-Württemberg } \\
{ }^{a} p=\text { signifikant } \\
{ }^{b} p=\text { höchstsignifikant }\end{array}$ & ganz zu", 5 , & nme überhaup & ot nicht zu" & & & \\
\hline
\end{tabular}

aufgrund des Verteilungsverfahrens durch verschiedene Praxispartner*innen,

- keine Vergleichs- und Verlaufsdaten von Hochschulbeschäftigten in Bezug auf Gesundheit und Wohlbefinden vor der Coronapandemie.

\section{Fazit für die Praxis}

- Die negativen sowie positiven Auswirkungen von Homeoffice/ Telearbeit und damit einhergehende Beanspruchungen müssen Beachtung im betrieblichen Gesundheitsmanagement von Hochschulen finden und in die psychische Gefährdungsbeurteilung aufgenommen werden, auch in Ausnahmesituationen.

- Die Gefährdungsbeurteilung gehört zum Aufgabebereich der Hoch- schulleitung. Weitere Akteure wie Betriebsärzt*innen, Fachkräfte für Arbeitssicherheit, Personalvertretung, Betriebliches Gesundheitsmanagement und Gleichstellung sollten einbezogen werden.

- Hochschulbeschäftigte mit Kind(ern) sollten beteiligt werden, um Bedarfe offenzulegen und zielgruppengerechte Maßnahmen, besonders beim Thema Entgrenzung, zu entwickeln.

- Wichtig ist eine offene und wertschätzende Kommunikation der Hochschulleitung für die Situation von Beschäftigten mit Kind(ern) auch nach der Pandemie.

\section{Korrespondenzadresse}

Prof. Dr. Marlen Niederberger

Forschungsmethoden in der Gesundheitsförderung und Prävention, Pädagogische Hochschule Schwäbisch Gmünd Oberbettringer Str. 200, 73525 Schwäbisch Gmünd, Deutschland

marlen.niederberger@ph-gmuend.de

Danksagung. Für die umfängliche Unterstützung danken wir unseren Partner*innen: Koordinierungsstelle Chancengleichheit Sachsen, Unfallkassen Baden-Württemberg und Sachsen, Landeskonferenz der Gleichstellungsbeauftragen an den wissenschaftlichen Hochschulen Baden-Württemberg (LaKoG), Karlsruher Institut für Technologie, Gesundheitsfördernde Hochschulen Südwest, Büro für Gleichstellung und Familie der Pädagogischen Hochschule Schwäbisch Gmünd und unseren Interviewpartner*innen.

Funding. Open Access funding enabled and organized by Projekt DEAL. 


\section{Einhaltung ethischer Richtlinien}

Interessenkonflikt. K. Allgayer, C. Bäßler, R. Jutz und M. Niederberger geben an, dass kein Interessenkonflikt besteht.

Für diesen Beitrag wurden von den Autoren keine Studien an Menschen oder Tieren durchgeführt. Für die aufgeführten Studien gelten die jeweils dort angegebenen ethischen Richtlinien.

Open Access. Dieser Artikel wird unter der Creative Commons Namensnennung 4.0 International Lizenz veröffentlicht, welche die Nutzung, Vervielfältigung, Bearbeitung, Verbreitung und Wiedergabe in jeglichem Medium und Format erlaubt, sofern Sie den/die ursprünglichen Autor(en) und die Quelle ordnungsgemäß nennen, einen Link zur Creative Commons Lizenz beifügen und angeben, ob Änderungen vorgenommen wurden.

Die in diesem Artikel enthaltenen Bilder und sonstiges Drittmaterial unterliegen ebenfalls der genannten Creative Commons Lizenz, sofern sich aus der Abbildungslegende nichts anderes ergibt. Sofern das betreffende Material nicht unter der genannten Creative Commons Lizenz steht und die betreffende Handlung nicht nach gesetzlichen Vorschriften erlaubt ist, ist für die oben aufgeführten Weiterverwendungen des Materials die Einwilligung des jeweiligen Rechteinhabers einzuholen.

Weitere Details zur Lizenz entnehmen Sie bitte der Lizenzinformation auf http://creativecommons.org/ licenses/by/4.0/deed.de.

\section{Literatur}

1. Barthel K (2020) Auswertung der Befragung aller Lehrenden der Beuth Hochschule im Sommersemester 2020. Beuth Hochschule, Berlin

2. Brakemeier E, Wirkner J, Knaevelsrud J, Wurm S, Christiansen H, Lueken U, Schneider S (2020) Die COVID-19-Pandemie als Herausforderung für die psychische Gesundheit Erkenntnisse und Implikationen für die Forschung und Praxis aus Sicht der Klinischen Psychologie und Psychotherapie. Z Klin Psychol Psychother. https://doi.org/10. 1026/1616-3443/a000574

3. Capitano J, Greenhaus JH (2018) When work enters the home: antecedents of role boundary permeability behavior. J Vocat Behav 109:87-100. https://doi.org/10.1016/j.jvb.2018.10.002

4. Deutsche Gesetzliche Unfallversicherung e.V. (2020) Psychische Belastung und Beanspruchung von Beschäftigten während der Coronavirus-Pandemie. https://publikationen.dguv.de/widgets/ pdf/download/article/3901. Zugegriffen: 18. Jan. 2020

5. Dragano N, Diebig M, Faller G, Honnings A, Hoven $H_{\text {, }}$ Lang J et al (2020) Arbeitsverdichtung, Angst vor Infektionen und Co.: Management psychischer Arbeitsbelastungen während der COVID-19 Pandemie. Gesundheitswesen 82(06):489-490. https://doi.org/10.1055/a-1172-2356

6. Frank R (2011) Fragebogen zum aktuellen körperlichen Wohlbefinden (FAW). In: Frank R (Hrsg) TherapiezielWohlbefinden, 2. Aufl.Springer, Berlin, Heidelberg, S 295-298

7. Gajendran RS, Harrison DA (2007) The good, the bad, and the unknown about telecommu- ting: meta-analysis of psychological mediators and individual consequences. J Appl Psychol 92(6):1524-1541. https://doi.org/10.1037/00219010.92.6.1524

8. Huebener M, Spieß CK, Siegel NA, Wagner GG (2020) Wohlbefinden von Familien in Zeiten von Corona: Eltern mitjungen Kindern am stärksten beeinträchtigt. DIW Wochenber 87(30/31):527-537. https://doi.org/10.18723/DIW_WB:2020-30-1

9. Kantar Public (2019) SOEP-Core - 2018: Personenfragebogen, Stichproben A-L3 + N. SOEP Survey Papers 608: Series A. DIW/ SOEP. https://www. diw.de/documents/publikationen/73/diw_01.c. 622046.de/diw_ssp0608.pdf.Zugegriffen: 16. Jan. 2021

10. Klonschinski A (2020) Der universitäre Mittelbau und die Corona-Pandemie. Deutsche Gesellschaft für Philosophie e. V. 48:1-10. https://dgphil.de/ fileadmin/newsletters/Mitteilungen-der-DGPhilNr-48.pdf. Zugegriffen:26. Jan. 2021

11. Kohlrausch B, Zucco A (2020) Die Corona Krise trifft Frauen doppelt: Weniger Erwerbseinkommen und mehr Sorgearbeit. Policy Brief WSI, 05/2020(40). https://www.wsi.de/de/faust-detail.htm?sync id=HBS-007676. Zugegriffen: 29. Juli 2021

12. Korbel JO, Stegle O (2020) Effects of the COVID19 pandemic on life scientists. Genome Biol 21(113):1-5. https://doi.org/10.1186/s13059020-02031-1

13. Lengen JC, Kordsmeyer A, Rohwer E, Harth V, Mache S (2020) Soziale Isolation im Homeoffice im Kontext der COVID-19-Pandemie. Hinweise für die Gestaltung von Homeoffice im Hinblick auf soziale Bedürfnisse. Zentralbl Arbeitsmed Arbeitsschutz Ergonomie. https://doi.org/10.1007/s40664-02000410-w

14. LötherA (2019) Hochschulranking nach Gleichstellungsaspekten 2019. (cews.publik, 23). Köln:GESIS - Leibniz-Institut für Sozialwissenschaften Fachinformation für die Sozialwissenschaften, Kompetenzzentrum Frauen in Wissenschaft und Forschung (CEWS). https://nbn-resolving.org/urn nbn:de:0168-ssoar-64113-9. Zugegriffen: 4. Dez. 2020

15. Mojtahedzadeh N, Rohwer E, Lengen J, Harth V, Mache S (2021) Gesundheitsfördernde Arbeitsge staltung im Homeoffice im Kontext der COVID19-Pandemie. Zentralbl Arbeitsmed Arbeitsschutz Ergonomie. https://doi.org/10.1007/s40664-02000419-1

16. Myers KR, Tham WY, Yin Y, Cohodes N, Thursby JG Thursby MC et al (2020) Unequal effects of the COVID-19 pandemic on scientists. Nat Hum Behav 4(9):880-883. https://doi.org/10.1038/s41562020-0921-y

17. Psychiatric Research Unit (WHO Collaborating Center for Mental Health, Frederiksborg General Hospital) (1998) WHO (Fünf) - Fragebogen zum Wohlbefinden. https://www.psykiatri-regionh. dk/who-5/Documents/WHO5_German.pdf. Zugegriffen:4.Dez. 2020

18. Robert Koch-Institut (2021) Gesamtübersicht der pro Tag ans RKI übermittelten Fälle, Todesfälle und 7-Tage-Inzidenzen nach Bundesland und Landkreis. https://www.rki.de/DE/Content/InfAZ/ N/Neuartiges_Coronavirus/Daten/Fallzahlen_ Daten.html.Zugegriffen: 29. März 2021

19. Sächsisches Staatsministerium für Soziales und Gesellschaftlichen Zusammenhalt (2020) Verordnung zum Schutz vor dem Coronavirus SARS-CoV-2 und COVID-19. https://www.coronavirus.sachsen. de/download/SMS-Saechsische-Corona-SchutzVerordnung-2020-12-11.pdf. Zugegriffen: 23. März 2021
20. Spinelli M, Lionetti F, Pastore M, Fasolo M (2020) Parents and childrenfacing the COVID-19 outbreak in Italy. Front Psychol 11:1713. https://doi.org/10. 3389/fpsyg.2020.01713

21. Staatsministerium Baden-Württemberg (2021) Orientierungshilfen zur Notbetreuung an den Kindertageseinrichtungen und in der Kindertagespflege. https://www.baden-wuerttemberg. de/fileadmin/redaktion/dateien/PDF/Anlagen_ PMs_2021/210106_Anlage_Orientierungshilfe Notbetreuung_Kita.pdf. Zugegriffen: 23. März 2021

22. Statistisches Bundesamt (2018) Arbeitsmarkt auf einen Blick. Deutschland und Europa. Wiesbaden: Statistisches Bundesamt. https:// www.destatis.de/Europa/DE/Publikationen/ Bevoelkerung-Arbeit-Soziales/Arbeitsmarkt/ broeschuere-arbeitsmark-blick-0010022189004. pdf?_blob=publicationFile. Zugegriffen: 20 . Jun 2021

23. Statistisches Bundesamt (2020) Pressemitteilung: Erwerbsbeteiligung von Müttern zwischen 2008 und 2018 bundesweit gestiegen. https://www. destatis.de/DE/Presse/Pressemitteilungen/2020/ 05/PD20_N023_132.html. Zugegriffen: 11. Juni 2021

24. Stifterverband für die Deutsche Wissenschaft e. V. (2020) Lage und Entwicklung der Hochschulen aus Sicht ihrer Leitungen. Hochschul-Barometer. Stimmungsbarometer | Bildung für Future Skills | Hochschulpolitik. https://www.stifterverband. org/download/file/fid/9483. Zugegriffen:23. März 2021

25. Van Der Feltz-Cornelis C, Varley D, Allgar V, De Beurs E (2020) Workplace stress, presenteeism, absenteeism and resilience amongst University staffand students in the COVID-19lockdown. Front Psychiatry 11:588803. https://doi.org/10.3389/ fpsyt.2020.588803

26. World Health Organization (1946) Constitution of the World Health Organization. World Health Organization, New York

27. Wydra G (2014) Der Fragebogen zum allgemeinen habituellen Wohlbefinden (FAHW und FAHW12): Entwicklung und Evaluation eines mehrdimensionalen Fragebogens, 5. Aufl. https://www. sportpaedagogik-sb.de/pdf/FAHW\%202020.pdf. Zugegriffen:6.Dez. 2020 